\title{
ON THE GENERAL SOLUTION OF A FUNCTIONAL EQUATION CONNECTED TO SUM FORM INFORMATION MEASURES ON OPEN DOMAIN - III
}

\author{
PL. KANNAPPAN and P.K. SAHOO \\ Faculty of Mathematics \\ University of Waterloo \\ Waterloo, Ontario, N2L 3GI
}

(Received August 6, 1985 and in revised form Apri1 30, 1986)

ABSTRACT. In this series, this paper is devoted to the study of a functional equation connected with the characterization of weighted entropy and weighted entropy of degree B. Here, we find the general solution of the functional equation ( 2 ) on an open domain, without using 0 -probability and 1-probability.

KEY WORDS AND PHRASES. Functional equation, weighted entropy, weighted entropy of degree B, open domain, sum form.

1980 MATHEMATICS SUBJECT CLASSIFICATION CODE: 94A15, 94A17, 39B40.

1. INTRODUCTION.

$$
\text { Let } \Gamma_{n}^{0}=\left\{P=\left(p_{1}, p_{2}, \ldots, p_{n}\right) \mid 0<p_{j}<1, \sum_{k=1}^{n} p_{k}=1\right\} \text { and } \Gamma_{n} \text { be the closure }
$$

of $\Gamma_{n}^{0}$. Let $\mathbb{R}_{+}=\{x \in \mathbb{R} \mid x>0\}$, where $\mathbb{R}$ is the set of real numbers. Let

$(\Omega, A, \mu)$ be a probability space and let us consider an experiment that is a finite measurable partition $\left\{A_{1}, A_{2}, \ldots, A_{n}\right\},(n>1)$ of $\Omega$. The weighted entropy of such an experiment is defined by Belis and Guiasu [1] as

$$
H_{n}^{1}(P, U)=-\sum_{k=1}^{n} u_{k} p_{k} \log p_{k}
$$

where $p_{k}=\mu\left(A_{k}\right)$ is the objective probability of the event $A_{k}$ ' $P=\left(p_{1}, p_{2}, \ldots, p_{n}\right) \in \Gamma_{n}$ and $U=\left(u_{1}, u_{2}, \ldots, u_{n}\right) \in \mathbb{R}_{+}^{n}$. The weigthed entropy of degree $B(B \in \mathbb{R}-\{1\})$ of an experiment is defined by Emptoz [2] as

$$
H_{n}^{B}(P, U)=\left(1-2^{1-\beta}\right)^{-1} \sum_{k=1}^{n} u_{k}\left(p_{k}-p_{k}^{B}\right) .
$$

The measures $H_{n}^{l}(P, U)$ satisfy the following functional equation (see Kannappan [3])

$$
\sum_{i=1}^{n} \sum_{j=1}^{m} f\left(p_{i} q_{j}, u_{i} v_{j}\right)=\sum_{i=1}^{n} p_{i} u_{j} \cdot \sum_{j=1}^{m} f\left(q_{j} \cdot v_{j}\right)+\sum_{j=1}^{m} q_{j} v_{j} \cdot \sum_{i=1}^{n} f\left(p_{i}, u_{i}\right)
$$

for all $P \in \Gamma_{n^{\prime}} Q \in \Gamma_{m^{\prime}} u_{i}, v_{j} \in \mathbb{R}_{+}$. A generalization of (1) is the following:

$$
\sum_{i=1}^{n_{1}} \sum_{j=1}^{m} f\left(p_{i} q_{j}, u_{i} v_{j}\right)=\sum_{i=1}^{n} p_{i}^{\alpha} u_{i} \cdot \sum_{j=1}^{m} f\left(q_{j}, v_{j}\right)+\sum_{j=1}^{m} q_{j}^{\beta} v_{j} \cdot \sum_{j=1}^{n} f\left(p_{i}, u_{i}\right),
$$


where $P \in \Gamma_{n}, Q \in \Gamma_{m^{\prime}}\left(u_{1}, u_{2}, \ldots, u_{n}\right) \in \mathbb{R}_{+}^{n},\left(v_{1}, v_{2}, \ldots, v_{m}\right) \in \mathbb{R}_{+}^{m}, \alpha, \beta \in \mathbb{R}-\{0,1\}$. The measurable solution of (1.2) for $\alpha=1$ was given by Kannappan in [3]. In a recent paper of Kannappan and Sahoo [4], measurable solution of a more general functional equation than (1.2) was given using the result of this paper. In this paper, we determine the general solution of (1.2) where $P \in \Gamma_{n^{\prime}}^{0} Q \in \Gamma_{m^{\prime}}^{0}\left(u_{1}, u_{2}, \ldots, u_{n}\right) \in \mathbb{R}_{+^{n}}^{n}$, $\left(v_{1}, v_{2}, \ldots, v_{m}\right) \in \mathbb{R}_{+}^{m}, \quad \alpha, \beta \in \mathbb{R}-\{0,1\}$ and $m, n$ (fixed and) $\geq 3$, on an open domain.

\section{SOLUTION OF (1.2) ON AN OPEN DOMAIN}

We need the following result in this sequel.

Result $1[5]$. Let $f, g:] 0,1[\rightarrow \mathbb{R}$ be real valued functions and satisfy

$$
\sum_{i=1}^{n} \sum_{j=1}^{m} f\left(p_{i} q_{j}\right)=\sum_{i=1}^{n} p_{i}^{\alpha} \cdot \sum_{j=1}^{m} g\left(q_{j}\right)+\sum_{j=1}^{m} q_{j}^{\beta} \cdot \sum_{i=1}^{n} f\left(p_{i}\right)
$$

for $P \in \Gamma_{n^{\prime}}^{0} Q \in \Gamma_{m^{\prime}}^{0}, \alpha, \beta \in \mathbb{R}-\{0.1\}$ and $m, n(\geq 3)$ are arbitrary but fixed integers. Then the general solutions of (2.1) are given by

$$
\left.\begin{array}{l}
f(p)=A(p)+a p^{\alpha}+b p^{\beta}, \\
g(p)=A^{\prime}(p)+a\left(p^{\alpha}-p^{\beta}\right)+c
\end{array}\right\} \text { for } \alpha \neq \dot{B}
$$

and

$$
\left.\begin{array}{l}
f(p)=A(p)+D(p) p^{\alpha}+d p^{\beta}, \\
g(p)=A^{\prime}(p)+D(p) p^{\alpha}+C
\end{array}\right\} \text { for } \alpha=B
$$

where $a, b, c, d$ are arbitrary constants, $A, A^{\prime}$ are additive functions on $\mathbb{R}$ with $A(1)=0, A^{\prime}(1)+m c=0$ and $D$ is a real valued function satisfying

$$
D(p q)=D(p)+D(q), \quad p, q \in] 0,1[.
$$

Now we proceed to determine the general solution of (1.2) on $] 0,1[$. Let $\mathrm{f}:] 0,1\left[\times \mathbb{R}_{+} \rightarrow \mathbb{R}\right.$ be a real valued function and satisfy the functional equation (1.2) for an arbitrary but fixed pair of positive integers $m, n(\geq 3)$, for $P \in \Gamma_{n^{\prime}}^{0}$ $Q \in \Gamma_{\mathrm{m}^{\prime}}^{0}$ with $\alpha, \beta \in \mathbb{R}-\{0,1\}$. Letting $u_{i}=u$ for all $i=1,2, \ldots, n$ and $v_{j}=v$ for $j=1,2, \ldots, m$ in (1.2), we obtain

$$
\sum_{j=1}^{n} \sum_{j=1}^{m} \frac{f\left(p_{i} q_{j}, u v\right)}{u v}=\sum_{i=1}^{n} p_{i}^{\alpha} \cdot \sum_{j=1}^{m} \frac{f\left(q_{j}, v\right)}{v}+\sum_{j=1}^{m} q_{j}^{\beta} \cdot \sum_{i=1}^{n} \frac{f\left(p_{i}, u\right)}{u},
$$

where $u, v \in \mathbb{R}_{+}$. Putting $v=1$ in $(2.3)$, we get

$$
\sum_{i=1}^{n} \sum_{j=1}^{m} \frac{f\left(p_{i} q_{j}, u\right)}{u}=\sum_{i=1}^{n} p_{i}^{\alpha} \cdot \sum_{j=1}^{m} f\left(q_{j}, 1\right)+\sum_{j=1}^{m} q_{j}^{\beta} \cdot \sum_{i=1}^{n} \frac{f\left(p_{i}, u\right)}{u}
$$

where $u, v \in \mathbb{R}_{+}$. Putting $v=1$ in $(2.3)$, we get

$$
\sum_{i=1}^{n} \sum_{j=1}^{m} \frac{f\left(p_{i} q_{j}, u\right)}{u}=\sum_{i=1}^{n} p_{i}^{\alpha} \cdot \sum_{j=1}^{m} f\left(q_{j}, 1\right)+\sum_{j=1}^{m} q_{j}^{B} \cdot \sum_{i=1}^{n} \frac{f\left(p_{i}, u\right)}{u}
$$

for $u \in \mathbb{R}_{+}$and $P \in \Gamma_{n^{\prime}}^{0} Q \in \Gamma_{m}^{0}$. For fixed $u \in \mathbb{R}_{+},(2.4)$ is of the form (2.1) and hence its general solutions cna be obtained from Result 1. 
First we consider the case $\underline{\alpha \neq \beta}$. Then from Result 1, we have

$$
\dot{f}(\mathrm{p}, \mathrm{u})=\mathrm{A}_{1}(\mathrm{p}, \mathrm{u}) \mathrm{u}+\mathrm{a}(\mathrm{u}) \mathrm{up}^{\alpha}+\mathrm{b}(\mathrm{u}) \mathrm{up}^{\beta}
$$

where $a, b: \mathbb{R}_{+} \rightarrow \mathbb{R}$ are real valued functions of $u$ and $A_{1}$ is additive in the first variable, with $A_{1}(1, u)=0$. Letting (2.5) into (2.3), we get

$$
\begin{aligned}
& (a(u v)-a(v)) \sum_{i=1}^{n} p_{i}^{\alpha} \sum_{j=1}^{m} q_{j}^{\alpha}+(b(u v)-b(u)) \sum_{i=1}^{n} p_{i}^{\beta} \cdot \sum_{j=1}^{m} q_{j}^{\beta} \\
& \quad-(b(v)+a(u)) \sum_{i=1}^{n} p_{i}^{\alpha} \cdot \sum_{j=1}^{m} q_{j}^{\beta}=0 .
\end{aligned}
$$

Noting $\alpha \neq B, \quad(\alpha \neq 1, \beta \neq 1)$ equating the coefficients of $\sum_{i=1}^{n} p_{i}^{\alpha}$ and $\sum_{i=1}^{n} p_{i}^{\beta}$ (then using the same for $\sum_{j=1}^{m} q_{j}^{\alpha}$ and $\left.\sum_{j=1}^{m} q_{j}^{\beta}\right)$ in (2.6), we get

$$
a(u v)=a(v), b(u v)=b(u) \text { and } b(v)=-a(u) .
$$

From these it is easy to see that

$$
a(u)=-b(v)=a, \text { constant }
$$

for all $u, v \in \mathbb{R}_{+}$. Now putting (2.7) into (2.5), we get

$$
f(p, u)=A_{1}(p, u) u+a u\left(p^{\alpha}-p^{B}\right)
$$

with $A_{1}(1, u)=0$. Again letting (2.8) into (1.2), we get

$$
\begin{aligned}
& \sum_{i=1}^{n} \sum_{j=1}^{m} A_{1}\left(p_{i} q_{j}, u_{i} v_{j}\right) u_{i} v_{j}=\sum_{j=1}^{m} A_{1}\left(q_{j}, v_{j}\right) v_{j} \sum_{i=1}^{n} u_{i} p_{i}^{\alpha}+ \\
& \quad+\sum_{i=1}^{n} A_{1}\left(p_{i}, u_{i}\right) u_{i} \cdot \sum_{j=1}^{m} v_{j} q_{j}^{B} .
\end{aligned}
$$

Since $A_{1}$ is additive in the first variable, by putting $u_{i}=1$ and $p_{i}=\frac{1}{n}$ (note that $\alpha \neq 1$ ), we have

$$
\sum_{j=1}^{m} A_{1}\left(q_{j}, v_{j}\right) v_{j}=0
$$

We let $v_{1}=v_{2}, \ldots,=v_{m-1}=v$ and $v_{m}=v^{\prime}$, where $v_{,} v^{\prime} \in \mathbb{R}_{+^{\prime}}$ into (2.10) and obtain

$$
\sum_{j=1}^{m-1} A_{1}\left(q_{j}, v\right) v+A_{1}\left(q_{m^{\prime}} v^{\prime}\right) v^{\prime}=0
$$

Since $A_{1}$ is additive in the first variable, and $A_{1}(1, v)=0$, we get

$$
A_{1}\left(q_{m}, v\right) v=A_{1}\left(q_{m^{\prime}}, v^{\prime}\right) v^{\prime}
$$

for all $\left.q_{m} \in\right] 0,1\left[\right.$, and $v, v^{\prime} \in \mathbb{R}_{+}$. From equation (2.11) it is clear that 


$$
A_{1}(x, y) y=A(x)
$$

where $A$ is an additive function with $A(1)=0$. Now using (2.12) in (2.8), we obtain

$$
\left.f(p, u)=A(p)+a u\left(p^{\alpha}-p^{\beta}\right), p \in\right] 0,1\left[, u \in \mathbb{R}_{+}\right.
$$

where $A$ is an additive function on $\mathbb{R}$ with $A(1)=0$ and $a$ is an arbitrary constant.

Next we consider the case $\underline{\alpha=B}$. Again the general solution of (2.4) from Result 1 can be obtained as

$$
f(p, u)=u A_{2}(p, u)+D_{1}(p, u) p^{\alpha} u+d(u) p^{\alpha} u
$$

where $d: \mathbb{R}_{+} \rightarrow \mathbb{R}$ is a real valued function of $u$ and $A_{2}$ is an additive function in the first variable with $A_{2}(l, u)=0$ and $\left.D_{1}:\right] 0,1\left[\times \mathbb{R}_{+} \rightarrow \mathbb{R}\right.$ satisfies (2.2). Putting (2.14) into (2.4), we get by equating the coefficient of $\sum_{i=1}^{n} p_{i}^{\alpha}$ (note $\alpha \neq 1$ )

$$
\sum_{j=1}^{m}\left[D_{1}\left(q_{j}, u\right)-D_{1}\left(q_{j}, 1\right)-d_{1}\right] q_{j}^{\alpha}=0 \text {. }
$$

Using $u=1$ in (2.15), gives $d_{1}=0$. Hence (2.15) with $d_{1}=0$, by the use of the Result 1 of [5], yields

$$
\left(D_{1}(x, u)-D_{1}(x, 1)\right) x^{\alpha}=A_{3}\left(x-\frac{1}{m}, u\right)
$$

for all $x \in] 0,1\left[\right.$ and $A_{3}$ is an additive function in the first variable. Since $D_{1}$ satisfies $(2.2)$, we get

$$
A_{3}\left(x-\frac{1}{m}, u\right) y^{\alpha}+A_{3}\left(y-\frac{1}{m}, u\right) x^{\alpha}=A_{3}\left(x y-\frac{1}{m}, u\right) \text {. }
$$

Putting $y=\frac{1}{m}$ and using $A_{3}(0, u)=0$ in $(2.17)$, we get

$$
A_{3}(x, u)=c_{1} A_{3}(1, u) \text {. }
$$

Since $A_{3}$ is additive in the first variable we obtain from (2.18) that $A_{3} \equiv 0$ for $x \in] 0,1\left[\right.$, and all $u \in \mathbb{R}_{+}$. Thus, (2.16) reduces to

$$
D_{1}(x, u)-D_{1}(x, 1)=0 \text {. }
$$

From (2.19), we see that $D_{1}$ is independent of $u$, i.e.

$$
\left.D_{1}(x, y)=D(x), \quad x \in\right] 0,1[
$$

and since $D_{1}$ satisfies (2.2), $D$ also satisfies (2.2). Using (2.20) in (2.14), we get

$$
f(p, u)=u A_{2}(p, u)+D(p) u p^{\alpha}+d(u) u p^{\alpha}
$$

where $A_{2}$ is additive with $A_{2}(1, u)=0$. Letting (2.21) into (2.3), we get

$$
(d(u v)-d(u)-d(v)) \sum_{i=1}^{n} \sum_{j=1}^{m}\left(p_{i} q_{j}\right)^{\alpha}=0
$$


for all $u, v \in \mathbb{R}_{+}$. Since $\sum_{i=1}^{n} \sum_{j=1}^{m}\left(p_{i} q_{j}\right)^{\alpha} \neq 0$ we obtain

$$
a(u v)=d(u)+d(v), \quad u, v \in \mathbb{R}_{+} \cdot
$$

Again putting (2.21) into (1.2) and using (2.23) and (2.2), we get

$$
\begin{aligned}
\sum_{i=1}^{n} \sum_{j=1}^{m} A_{2}\left(p_{i} q_{j}, u_{i} v_{j}\right) u_{i} v_{j}=\sum_{i=1}^{n} u_{i} p_{i}^{\alpha} \cdot \sum_{j=1}^{m} A_{2}\left(q_{j}, v_{j}\right) v_{j}+ & \\
& +\sum_{j=1}^{m} v_{j} q_{j}^{\alpha} \cdot \sum_{i=1}^{n} A_{2}\left(p_{i}, u_{i}\right) u_{i} \cdot \\
\text { Putting } u_{i}= & 1 \text { and } p_{i}=\frac{1}{n} \text { in }(2,4) \text {, we obtain } \\
& \sum_{j=1}^{m} A_{2}\left(q_{j}, v_{j}\right) v_{j}=0 .
\end{aligned}
$$

Note that $(2.25)$ is of the form of (2.10) and hence by a similar argument we get

$$
A_{2}(q, u) u=A(q)
$$

where $A$ is additive with $A(1)=0$. Using (2.26) in (2.21), we obtain

$$
f(p \cdot u)=A(p)+D(p) u p^{\alpha}+d(u) u p^{\alpha}
$$

where $A$ is additive on $\mathbb{R}$ with $A(1)=0$ and $D:] 0,1\left[\rightarrow \mathbb{R}, d: \mathbb{R}_{+} \rightarrow \mathbb{R}\right.$, are functions satisfying $(2.2)$ and $(2.23)$ respectively.

Thus we have proved the following theorem.

Theorem. Let $f:] 0,1\left[\times \mathbb{R}_{+} \rightarrow \mathbb{R}\right.$ be a real valued function satisfying (1.2) for arbitrary but fixed pair of $\mathrm{m}, \mathrm{n}(\geq 3)$ and $\alpha, \beta \notin\{0,1\}, P \in \Gamma_{\mathrm{n}^{\prime}}^{0} Q \in \Gamma_{\mathrm{m}}^{0}$. Then $\mathrm{f}$ is given by (2.13) when $\alpha \neq \beta$ and by (2.27) when $\alpha=\beta$.

Corollary. If $f$ is measurable in the Theorem then

$$
f(p, u)=a\left(p^{\alpha}-p^{\beta}\right) \quad \alpha \neq \beta
$$

and

$$
f(p, u)=\operatorname{bup}^{\alpha} \log p+c p^{\alpha} u \log u, \alpha=\beta
$$

where $a, b, c$ are arbitrary constants.

Remark. Because of the occurrence of the parameters $\alpha, \beta$ as powers, $f$ is independent of $m$ and $n$.

ACKNOWLEDGEMENT. This work is partially supported by a NSERC of Canada grant.

\section{REFERENCES}

1. BELIS, M. and GUIASU, S.; A quantitative-qualitative measure of information in cybernetic systems, IEEE Trans. Inform. Theory 1T-14, 593-594 (1968).

2. EMPTOZ, H.; Information de type $B$ integrant un concept d'utilite, C.R. Acad. Sci. Paris 282 A, 911-914 (1976). 
3. KANNAPPAN, PL.; On some functional equations from additive and nonadditive measures - III, Stochastica, 4, 15-22 (1980).

4. KANNAPPAN, PL. and SAHOO, P.K.; On a functional equation connected to sum form nonadditive information measures on open domain - III, to appear in Stochastica, (1985).

5. KANNAPPAN, PL. and SAHOO, P.K.; On the general solution of a functional equation connected to sum form information measures on open domain - II, (submitted), 1985. 


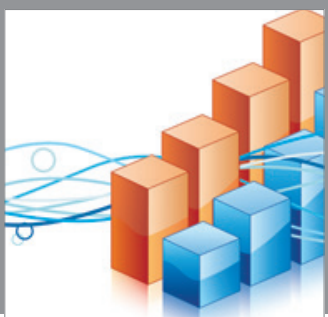

Advances in

Operations Research

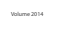

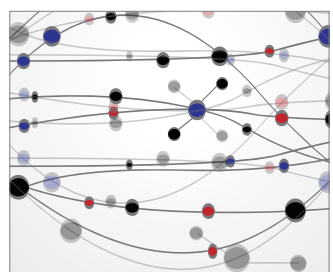

\section{The Scientific} World Journal
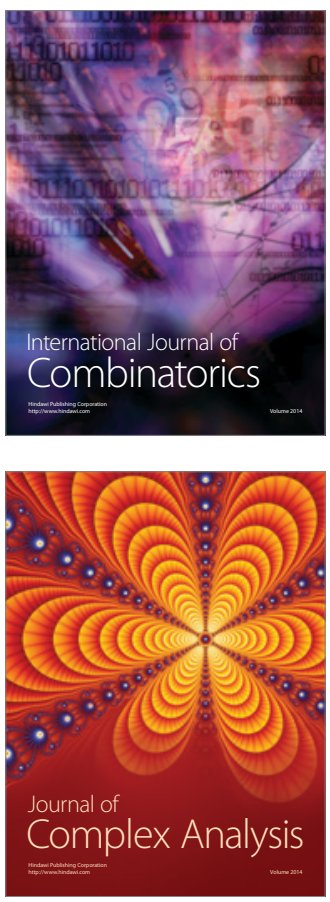

International Journal of

Mathematics and

Mathematical

Sciences
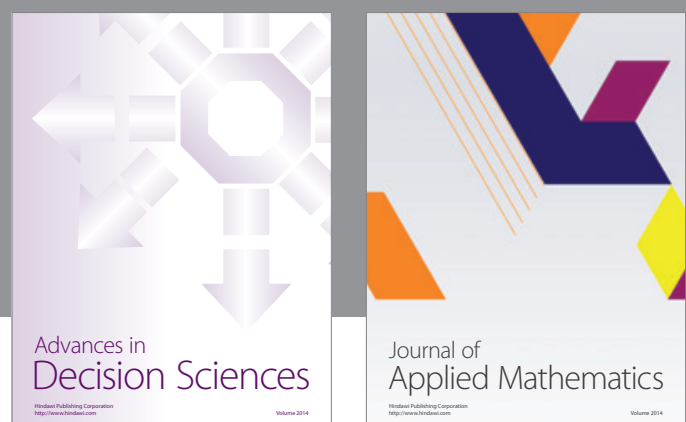

Journal of

Applied Mathematics
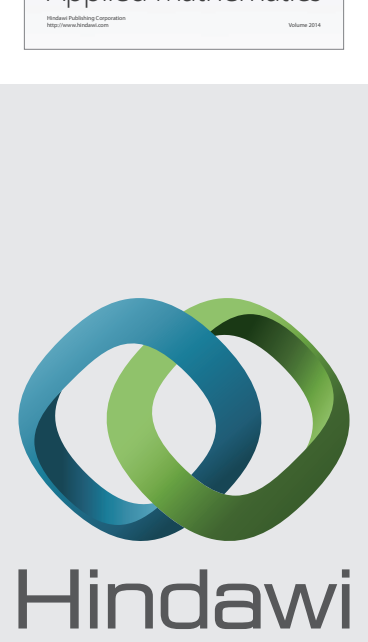

Submit your manuscripts at http://www.hindawi.com
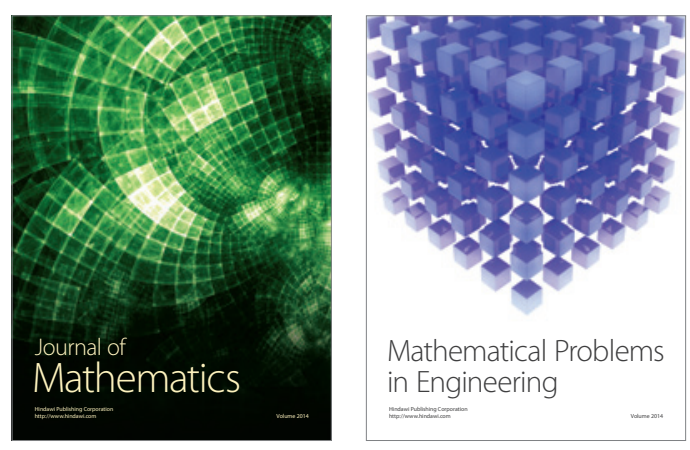

Mathematical Problems in Engineering
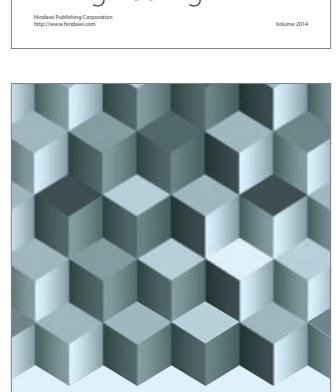

Journal of

Function Spaces
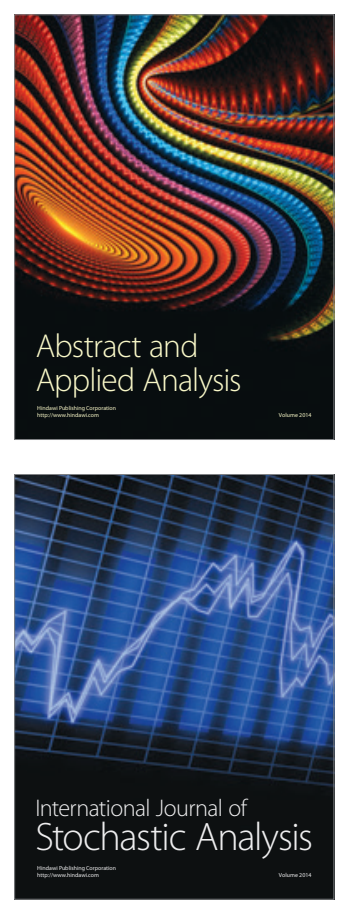

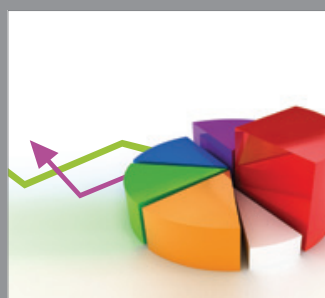

ournal of

Probability and Statistics

Promensencen
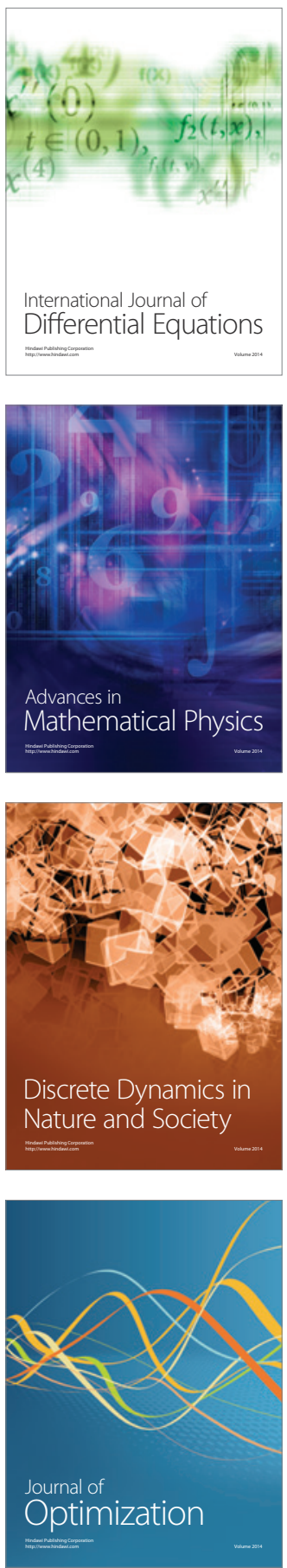